\title{
Correction to: A Novel Total Drug Assay for Quantification of Anti-C5 Therapeutic Monoclonal Antibody in the Presence of Abundant Target
}

Hiroo Watanabe, ${ }^{1,3}$ Mitsuko Shibuya, ${ }^{1}$ Norihito Shibahara, ${ }^{1}$ Yoshinao Ruike, ${ }^{2}$ Zenjiro Sampei, ${ }^{1}$ Kenta Haraya, ${ }^{1}$ Tatsuhiko Tachibana, ${ }^{1}$ Tetsuya Wakabayashi, ${ }^{1}$ Akihisa Sakamoto, ${ }^{1}$ Hiroyuki Tsunoda, ${ }^{2}$ and Naoaki Murao ${ }^{1}$

Correction to: The AAPS Journal volume 23, Article number: 21 (2021) https://doi.org/10.1208/s12248-020-00539-9

A formatting error occurred on Table IV during production. The original article has been corrected.

Publisher's Note Springer Nature remains neutral with regard to jurisdictional claims in published maps and institutional affiliations.

The online version of the original article can be found at https:// doi.org/10.1208/s12248-020-00539-9

\footnotetext{
${ }^{1}$ Research Division, Chugai Pharmaceutical Co., Ltd., 1-135 Komakado, Gotemba, Shizuoka 412-8513, Japan.

${ }^{2}$ Research Division, Chugai Pharmaceutical Co., Ltd., 200 Kajiwara, Kamakura, Kanagawa 247-8530, Japan.

${ }^{3}$ To whom correspondence should be addressed. (e-mail: watanabehro@chugai-pharm.co.jp)
} 